\section{Preparing Emergency Medical Services for World Motor-Sporting Events: Experiences at the World Motorcycle Grand Prix and the Formula-One World Championships 1999, Sepang International Circuit, Malaysia \\ Dr. A. Abdul Aziz,; Col. (Dr) MZ Bidin, ${ }^{2}$ Col. (Dr) S. Abdullab ${ }^{3}$}

1) Emergency Physician, National University Hospital of Malaysia, Kuala Lumpur; 2) Commanding Officer, Army Hospital, Trendak Camp, Malacca; 3) Commanding Officer, Army Hospital, Kinrara Camp, Kuala Lumpur, MALAYSIA

Malaysia experienced its first ever opportunity of hosting two world motor-sporting events in 1999: the World Motorcycle Grand Prix and the Formula-One in April and October respectively. The venue was a newly built motor circuit in Sepang that has a Grandstand capacity of 30,000 and open-air ground seating capacity of around 100,090 . Included in the set-up was a well-designed medical centre equipped with resuscitation, radiology, and operating theatre services.

The emergency medical services for both events were planned and provided by the Malaysian Armed Forces Health Services with immediate support from the National University Hospital of Malaysia, the nearest and most advanced tertiary emergency and trauma centre. With a decade's experience organising local motorcycle grand prix but none for the Formula One Championships, planning and preparations for these world-class events at the brand new circuit, however, were extensive.

This paper presents the preparations undertaken and challenges encountered, with a view to sharing our experiences and assisting other organisations in preparing for similar motor-sporting events.

Keywords: emergency medical services; Formula One; grand prix; management; motorcycle; motorsports; preparedness

E-mail: azhar@hukm.ukm.my

\section{Friday, 29, September. 2000 Plenary Session}

Disaster Management Evaluation: The Current Status of the Standardised Protocol in the Utstein Style KO Sundnes, $M D$

Task Force on Quality Control of Disaster Management (TFQCDM), NORWAY

Since the first publication as a Disaster Research Template in 1996, ${ }^{1}$ its refinement has progressed substantially. The process used and the product itself have been presented at different workshops and congresses, including the previous Nordic Congress in Kuopio. ${ }^{2}$ This process became necessary in order to widen the scope for feedback and discussion during its development and to ensure its long-term dissemination. The process now is formally tied to the World Association of Disaster and Emergency Medicine (WADEM), since the General Assembly at the World Congress in Osaka in 1999 endorsed the Executive Summary. ${ }^{3}$ This summer, the World Health Organisation (Department for Emergency and Humanitarian Action) has signalled a wish to participate more actively, both in the ongoing development and also in the active use of the protocol as basis for research and teaching. The Task Force on Quality Control of Disaster Management (TFQCDM) remains, however, an independent body as previously outlined. $^{2}$

For comparative research, disasters will be divided into identifiable phases, each described exclusively by its properties and not by time. It should facilitate comparisons regardless of the type of event that is responsible for the disaster. For example, the impact phase may vary from seconds (earthquakes) to weeks (flooding) to years (droughts), but still is the same phase. Furthermore, any or all of the Basic Societal Functions that together constitute a society may be followed through each of the defined phases of disaster, as well as how the functional status of each changes, depending on the kind of assistance provided or not provided. In this context the concept of "Best Outcome Without Assistance" (BOWA) will become crucial. $^{3}$

Since the previous presentation at the 11th. World Congress on Disaster and Emergency Medicine in Osaka in 1999, further elements and concepts have been discussed in more detail. The formula that can be used to analyse all of the elements that lead to a disaster has been refined to provide a better instrument for achieving an understanding of the elements that together constitute the Damage Probability. The ultimate goal of the project, is to identify and modify the key factors responsible for turning an event into a disaster.

The Guidelines provide the instruments required to analyse not only how a disaster is handled, but also how pre-event activities may prove crucial to reduce damage in a cost-beneficial way. To analyse is to evaluate. To evaluate is to attribute a value.

We need to identify proper sets of Indicators of Effectiveness so that each activity can be performed with 
utmost efficiency. Different activities then will be compared as to which is more effective in solving a problem. However, we still will need to define a different set of values if we want to verify if an activity really has benefited the victims. This demands a comprehensive understanding of the use of Indicators of Effectiveness, since one commodity (or action) may serve both as a variable and a parameter. (Water may serve as a parameter with regard to logistics, but would be a variable with regard to Crude Mortality Rate in a refugee camp). To some extent, the indicators will be quantitative, to some extent qualitative.

The TFQCDM has developed the indicators needed for the computations of a Disaster Severity Score and a Health Disaster Severity Score. Also, the concept of a Vulnerability-Preparedness Index will be subjected to already identified indicators. However, a complete list of Indicators of Effectiveness must be developed as the Guidelines become used more widely. All in all, the proper use of the Disaster Research Template should provide all of the elements needed to finally start to analyse disasters, their potential for reduction, and their management in an institutionalised way.

\section{References}

1. TFQCDM: Disaster medical response research: A template in the Utstein style. Prehosp Disast Med 1996:11;16-24.

2. Sundnes KO: Evaluation of health disaster management. Prehosp Disast Med 1998:13;S9. Abstract

3. TFQCDM, Chair: Sundnes KO: Health disaster management: Guidelines for evaluation and research in the Utstein style: Executive summary. Prebosp Disast Med 1999:14;43-52.

Keywords : basic elements; BOWA; damage probability; disaster medicine; evaluation; qualitative methods; quantitative methods; research; severity scores; template; vulnerability-preparedness index

\section{Theme 5. Application of International Standards to Disasters}

Chairs: Dr. Frederick Burkle $\sigma^{\circ}$ Dr. Judy Isaac-Renton

\section{Standards of Care in a Crisis Environment: Lessons Learned from Complex Emergencies \\ Frederick $M$. Burkle, Jr., $M D, M P H$}

University of Hawaii John A. Burns School of Medicine, Honolulu, Hawaii, USA

Nation-state internal conflicts, caused primarily by political factors, have resulted in high levels of violence primarily in vulnerable population groups of children, females, and the elderly, and in the loss of sustainable livelihood. A major characteristic of these complex emergencies is that they represent catastrophic public health emergencies.

The international humanitarian community entered the decade of the 1990s with little expertise and no standards of care. Parameters for water, nutrition, sanitation, communicable diseases, shelter, essential drugs, and health and nutrition assessments were developed with both painstaking trial and error and focused field and outcome research. By the end of the decade, the International Federation of Red Cross and Red Crescent (LFRC) had developed the Sphere Project to standardize care and codes of conduct, the UN Agencies had developed training manuals and guidelines, and University-NGO partnerships offered education, training, and research opportunities to further professionalize the disaster and humanitarian response.

Similar efforts are required to develop operational standards of care for reproductive health and women's issues and mental health interventions. Organizational management standards are lacking in the areas of information technologies and sharing, security, coordinated logistics, measures of effectiveness, and civil-military collaboration.

Keywords: complex emergencies; disasters; education; humanitarian; management; research; responses; standards; training 